\title{
Valorización de residuos proveniente del sector frutihortícola para la producción de productos químicos intermedios y biocombustibles
}

\section{Recovery of waste from the fruit and vegetable sector for the production of intermediate chemical products and biofuels}

Presentación: 6-7/10/20

\section{Doctorando:}

\section{Nicolás Guiñazú}

Grupo Pirólisis, Centro de Investigación y Tecnología Química, Facultad Regional Córdoba, Universidad Tecnológica Nacional- Argentina Nicolas.guiazu@gmail.com

\section{Director/a:}

\section{Horacio Falcón}

\section{Co-director/a:}

\section{Soledad Renzini José Antonio Alonso}

\section{Resumen}

En la presente tesis se investigará la producción de biolíquido obtenido mediante la pirólisis rápida de residuos agroindustriales provenientes del procesamiento de frutas, sobre un sistema catalítico compuesto por un material con estructura del tipo pirocloro $\left(\mathrm{H}_{3} \mathrm{O}\right) \mathrm{SbTeO}_{6}$. Las propiedades más relevantes del material catalítico para el proceso serán estudiadas por XRD, FTIR y SEM. La caracterización de la biomasa será realizada mediante análisis termogravimétrico (TG-DTG). La pirólisis rápida de pulpa de pera, cáscara de membrillo y fibra de batata se realizará a temperaturas entre 300 y $450 \stackrel{\circ}{\circ}$ en un lecho fijo durante 20 minutos, bajo atmósfera de $\mathrm{N}_{2}$ y en condiciones de vacío. El análisis de los productos de reacción obtenidos se llevará a cabo en un cromatógrafo de gases acoplado a espectrometría de masas (CGEM).

Palabras clave: Pirólisis; Biomasa; Residuos frutihortícolas; Catalizadores

\begin{abstract}
In this thesis, the production of bioliquid obtained by fast pyrolysis of agroindustrial residues from fruit processing will be investigated, on a catalytic system composed of a material with a pyrochloro type structure $\left(\mathrm{H}_{3} \mathrm{O}\right) \mathrm{SbTeO}_{6}$. The most relevant properties of the catalytic material for the process will be studied by XRD, FTIR and SEM. The characterization of the biomass will be carried out by thermogravimetric analysis (TG-DTG). The fast pyrolysis of pear pulp, quince peel and sweet potato fiber will be carried out at temperatures between 300 and $450{ }^{\circ} \mathrm{C}$ in a fixed bed for 20 minutes, under a $\mathrm{N}_{2}$ atmosphere and under vacuum conditions. The analysis of the obtained reaction products will be carried out in a gas chromatograph coupled to mass spectrometry (GC-MS).
\end{abstract}

Keywords: Pyrolysis; Biomass; Fruit and vegetables waste; Catalysts 


\section{Introducción}

La biomasa ha sido motivo de numerosos estudios, al ser considerada como una prometedora fuente sostenible de energía renovable, la cual permite minimizar los riesgos ambientales (Brosowski et al., 2016; Ghosh, 2016; Mamvur et al., 2020; Malico et al., 2019; Jianga et al., 2017). Los residuos sólidos orgánicos pueden ser procesados en presencia de oxígeno o en su ausencia mediante la digestión anaeróbica. Ambos métodos producen un efecto acondicionador de suelos, una especie de abono o fertilizante, que si se prepara correctamente también puede ser utilizado como una valiosa fuente de nutrientes en la agricultura urbana. En la digestión anaerobia también se produce un biogás, principalmente metano, una importante fuente de bioenergía (Sarker, 2020; Bedoi et al., 2019). Sin embargo, la generación de energía utilizando un biocombustible gaseoso, tiene limitaciones significativas en relación con los requisitos de manipulación de materiales y con respecto a su eficiencia energética. Una tecnología para la producción de combustibles líquidos, compuestos químicos de mayor valor agregado y biocarbón, es la pirólisis de biomasa (Kaczor et al., 2020; Persson et al., 2019; Zheng et al., 2020; Wua et al., 2019; Klinger et al., 2018). La pirólisis es un proceso termoquímico mediante el cual se convierte la biomasa de residuos sólidos en tres productos: un líquido, coque y un gas de efecto invernadero neutro. La pirólisis es una descomposición que ocurre en ausencia de oxígeno. El proceso tiene tres etapas: la dosificación y alimentación de la materia prima, la transformación de la masa orgánica y, finalmente, la obtención y separación de los productos (coque, bioaceite y gas) (Soosan et al., 2020; Casoni et al., 2016; Samolada et al., 2000; Aysu, 2015; Mi-Jin et al., 2013).

En la tesis se propone introducir una variante a esta tecnología térmica mediante la incorporación de materiales catalíticos que permitan la obtención de un bioaceite con una mayor densidad energética, productos químicos plataformas y biocarbón a partir de residuos de la industria frutihortícola. Asimismo, se propone promover el establecimiento de alianzas público-privadas que encaren la aplicación de esta tecnología y el entrenamiento de recursos humanos en el área del procesamiento de residuos de esta índole.

\section{Desarrollo}

\section{Síntesis y caracterización de catalizadores}

A) Desarrollo de nuevos catalizadores ácidos basados en la estructura tipo pirocloro, $\mathrm{A}_{2} \mathrm{~B}_{2} \mathrm{O}_{6} .(\mathrm{A}=\mathrm{K}, \mathrm{y} \mathrm{B}=\mathrm{Sb}, \mathrm{Te}, \mathrm{Mo}$, $\mathrm{Nb}, \mathrm{W})$

Los materiales laminares con estructura de pirocloro se llevarán a cabo con un procedimiento que consiste básicamente en la obtención en primer lugar del pirocloro de potasio $\mathrm{K}_{2} \mathrm{~B}_{2} \mathrm{O}_{6}(\mathrm{~B}=\mathrm{Sb}, \mathrm{Te}, \mathrm{Mo}, \mathrm{Nb}, \mathrm{W})$ por reacción de estado sólido y luego tratado en ácido sulfúrico concentrado para que intercambie los $\mathrm{K}+$ por $\mathrm{H} 30+$. Se intentará desviarse de la estequiometría 1:1 en la posición B, para alojar cantidades variables de $\mathrm{H}_{3} \mathrm{O}^{+}$.

B) Síntesis de zeolitas microporosos de poro medio del tipo ZSM-11

Se emplearán técnicas de síntesis para los materiales propuestos del tipo hidrotérmicas. Se pondrá especial énfasis en los tratamientos térmicos post-síntesis. Se incorporarán diferentes funciones activas a la matriz zeolítica tales como H, Zn, $\mathrm{Ni}$, entre otros. El dopaje de estas se realizará por impregnación vía húmeda o intercambio iónico a partir de sus sales correspondientes (acetatos, nitratos, cloruros). Las formas NH4-Zeolitas serán puestas en contacto con las soluciones acuosas de las sales correspondientes con la cantidad de metal que se desea incorporar sobre la matriz en un evaporador rotatorio al vacío hasta que la muestra se encuentre seca. Se estudiará el grado de incorporación de los cationes controlando: tipo de fuente precursora del elemento (el tipo de sal empleada influye en la incorporación de las especies), $\mathrm{pH}$ de las soluciones (es clave para evitar que se formen precipitados de compuestos formados por los metales y no intervengan en el proceso de incorporación en el soporte) y temperaturas (influye con características semejantes a las variantes antes mencionadas), etc. 


\section{Caracterización de los residuos frutihortícolas}

Para determinar la composición relativa de la biomasa: lignina, celulosa y hemicelulosas, se realizarán análisis termogravimétricos en una termobalanza modelo TGA /SDTA851e/SF $/ 1100^{\circ} \mathrm{C}$ Mettler Toledo. Se estudiará el comportamiento térmico de las distintas biomasas, en atmósfera inerte desde temperatura ambiente hasta $700{ }^{\circ} \mathrm{C}$. La pérdida de peso y la velocidad de calentamiento se registrarán continuamente a lo largo del experimento.

\section{Sistema de pirólisis}

La pirólisis de los residuos se llevará a cabo en un reactor de cuarzo de lecho fijo horizontal a escala de laboratorio, ubicado en el interior de un horno, como se esquematiza en la Figura 1.

La muestra sólida se colocará en una cápsula de cerámica y se introducirá en el reactor tubular de cuarzo por medio de un brazo de hierro móvil.

La introducción de la cápsula a la zona de pirólisis se realizará una vez logradas todas las condiciones de reacción como presión, temperatura y caudal de gas transportador. El extremo opuesto al ingreso de la muestra el reactor estará conectado a una bomba de vacío, logrando tiempos de residencia muy cortos $(<1 \mathrm{~s}$ ) de los productos formados a altas temperaturas. Una vez que los productos primarios emerjan de la zona de pirólisis, serán atrapados a temperaturas criogénicas (-200 a $\left.-170^{\circ} \mathrm{C}\right)$ evitando reacciones secundarias indeseables. El flujo de gas transportador en el sistema, generalmente nitrógeno ultra seco gaseoso, ayudará a la transferencia de los productos formados a altas temperaturas hacia la zona de condensación favoreciendo los procesos unimoleculares.

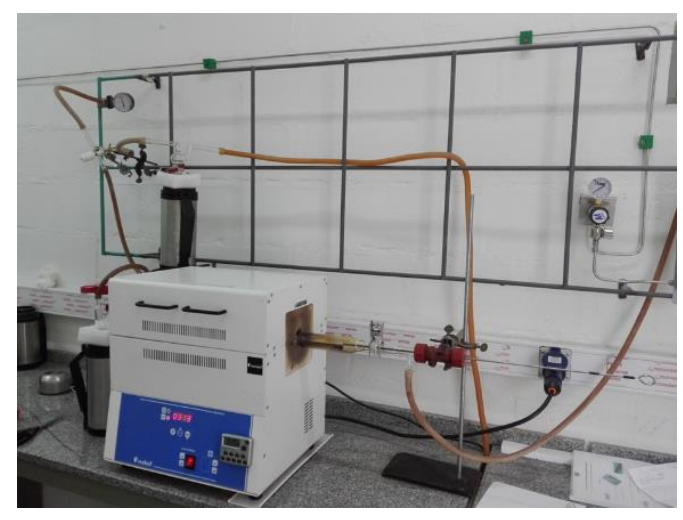

Figura 1. Sistema de pirólisis catalítica

En primer lugar se pesará aproximadamente $1 \mathrm{~g}$ de biomasa seca y se adicionará una determinada cantidad de catalizador ácido (5 - 2,5 \%P/P). Ambos sólidos se machacarán hasta obtener una mezcla homogénea. Se colocará la mezcla en la cápsula de cerámica y se introducirá en el reactor. Se trabajará a temperaturas entre 300 y $450{ }^{\circ} \mathrm{C}$, presiones de 50 mTorr y flujo de $\mathrm{N} 2$ de $0,2 \mathrm{~mL} / \mathrm{s}$. En estas condiciones, se introducirá la cápsula dentro de la zona de calentamiento del reactor y el tiempo de reacción será de 20 minutos. Transcurrido ese tiempo, se enfriará y retirará la cápsula y la trampa colectora de líquido. 
El biolíquido se extraerá de la trampa de condensación con acetona y se utilizará un evaporador rotatorio para separar el solvente de la muestra. La composición química de los biolíquidos se determinará mediante cromatografía gaseosa en un equipo Varian 3800 acoplado con un espectrómetro de masas. La caracterización de los productos se realizará por comparación con la biblioteca NIST considerando un match superior a 80 \%. Para cada reacción, se establecerán las cantidades de fracción líquida y sólida por peso directo y se calculará la fracción gaseosa por diferencia.

Al residuo carbonoso, obtenido a mayores temperaturas, más rico en carbón se lo tratará adecuadamente para generar carbón activado que podrá ser utilizado para remediación de aguas residuales y como enmienda de suelos de las huertas que proveen los frutos destinados a la manufactura de mermeladas y derivados.

\section{Conclusiones}

Se buscará, a lo largo del desarrollo de mi tesis doctoral, desarrollar una tecnología que contribuya a mejorar la sustentabilidad económica y ambiental de la agroindustria frutihortícola mediante la valorización de los residuos que generan, focalizando las acciones hacia el desarrollo de soluciones tecnológicas factibles de ser adoptadas por el sector empresarial. Las empresas del sector tendrán una herramienta estratégica que les permitirá un mejor posicionamiento competitivo en el mercado, influyendo en la capacidad de generar empleo.

\section{Referencias}

Aysu, T. (2015). Catalytic pyrolysis of Alcea pallida stems in a fixed-bed reactor for production of liquid bio-fuels. Bioresource Technology 191, 253-262. https://doi.org/10.1016/j.biortech.2015.05.037

Bedoi, R., Cucek, L., Cosi, B., Krajn, D., Smoljani, G., Kravanj, Z., Ljubas, D., Puksec, T., Duic, N. (2019). Green biomass to biogas e A study on anaerobic digestion of residue grass. Journal of Cleaner Production 213, 700-709. https://doi.org/10.1016/j.jclepro.2018.12.224 0.

Brosowski, A., Thran, D., Mantau, U., Mahro, B., Erdmann, G., Adler, P., Stinnera, W., Reinhold, G., Hering, T., Blanke, C. (2016). A review of biomass potential and current utilisation e Status quo for 93 biogenic wastes and residues in germany. Biomass and Bioenergy, 95, 257-272. http://dx.doi.org/10.1016/j.biombioe.2016.10.017

Casoni, A.I., Nievas, M.L., Moyano, E.L., Álvarez, M., Diez, A., Dennehy, M., Volpe, M.A. (2016). Catalytic pyrolysis of cellulose using MCM-41 type catalysts. Applied Catalysis A: General 514, 235-240. https://doi.org/10.1016/j.apcata.2016.01.017

Ghosh, S.K. (2016). Biomass \& Bio-waste Supply Chain Sustainability for Bioenergy and Bio-fuel Production. Procedia Environmental Sciences, 31, 31-39. doi: 10.1016/j.proenv.2016.02.005

Jianga, Y., van der Werf, E., van Ierland, E.C., Keesman, K.J. (2017). The potential role of waste biomass in the future urban electricity system. Biomass and Bioenergy 107, 182-190. http://dx.doi.org/10.1016/j.biombioe.2017.10.001

Kaczor, Z., Bulinski, Z., Werle, S. (2020). Modelling approaches to waste biomass pyrolysis: a review. Modelling approaches to waste biomass pyrolysis: a review. Renewable Energy, 159, 427-443. https://doi.org/10.1016/j.renene.2020.05.110.

Klinger, J.L., Westover, T.L., Emerson, R.M., Williams, C.L., Hernandez, S., Monson, G.D., Ryan, J.C. (2018). Effect of biomass type, heating rate, and sample size on microwave enhanced fast pyrolysis product yields and qualities. Applied Energy, 228, 535-545. https://doi.org/10.1016/j.apenergy.2018.06.107. 
Malico, I., Pereira, R.N., Gonçalves, A.C., Sousa, A.M.O. (2019). Current status and future perspectives for energy production from solid biomass in the European industry. Renewable and Sustainable Energy Reviews, 112, 960977. https://doi.org/10.1016/j.rser.2019.06.022

Mamvur, T.A., Danh, G. (2020). Biomass torrefaction as an emerging technology to aid in energy production. Heliyon, 6 , 3531. https://doi.org/10.1016/j.heliyon.2020.e03531.

Mi-Jin, J., Jong-Ki, J., Dong, J.S., Sung H.P., Young, J.S., Sang, H.J., Young-Kwon, P. (2013). Catalytic pyrolysis of biomass components over mesoporous catalysts using Py-GC/MS. Catalysis Today 204, 170- 178. https://doi.org/10.1016/j.cattod.2012.07.039

Persson, H., Yang, W. (2019). Catalytic pyrolysis of demineralized lignocellulosic biomass. Fuel, 252, 200-209. https://doi.org/10.1016/j.fuel.2019.04.087.

Samolada, M.C., Papafotica, A., Vasalos, I. A. (2000). Catalyst Evaluation for Catalytic Biomass Pyrolysis. Energy \& Fuels, 14, 1161-1167. https://doi.org/10.1021/ef000026b

Sarker, S. (2020). Exploring biogas potential data of cattle manure and olive cake to gain insight into farm and commercial scale production. Data in Brief 32, 106045. https://doi.org/10.1016/j.dib.2020.106045.

Soosan, K., Younghyun, L., Kun-Yi, A.L., Eunmi, H., Eilhann, E.K., Jechan, L. (2020). The valorization of food waste via pyrolysis. Journal of Cleaner Production 259. https://doi.org/10.1016/j.jclepro.2020.120816.

Wua, Z., Lia, Y., Zhaob, J., Zhanga, B., Xub, D., Yang, B. (2019). Fast pyrolysis behavior of lignocellulosic biomass model compound: releasing properties, kinetic analysis of the primary gaseous products and char structure evolution from cellulose. Energy Procedia, 158, 79-84. https://doi.org/10.1016/j.egypro.2019.01.049.

Zheng, A., Huang, Z., Wei, G., Zhao, K., Jiang, L., Zhao, Z., Tian, Y., Li H. (2020). Controlling Deoxygenation Pathways in Catalytic Fast Pyrolysis of Biomass and Its Components by Using Metal-Oxide Nanocomposites. iScience, 23, 100814. https://doi.org/10.1016/ j.isci.2019.100814. 